\title{
Agent-Based Evolving Societies
}

\author{
Loïs Vanhée ${ }^{1,2}$, Jacques Ferber ${ }^{1}$, Frank Dignum ${ }^{2}$ \\ ${ }^{1}$ LIRMM, University of Montpellier II, France \\ ${ }^{2}$ Utrecht Universiteit, The Netherlands
}

\begin{abstract}
This paper describes a method to build artificial societies that are capable of expanding themselves from bottom-up in order to adapt to changes occurring in the environment. These changes trigger social issues at the individual level which are reported at the global level. Then, the society expands itself with an organization enforcing individual behavior that copes with the issue.

We apply this method to model the first stages of human societies. These stages are confronted with dramatic changes in the population size. These changes lead to the creation of social-control organizations to face the evolution from a familial tribe, to an autocratic chiefdom to finally reach a bureaucratic state.
\end{abstract}

Keywords: Methodologies for MABS, Social Simulation, Simulating Social Complexity

\section{Introduction}

How can a society become aware of global problem and decide to solve it from bottom up? A simple solution consists in expanding itself with new organizations. Thus, firehouses are created to solve the problem of frequent fires. But, these firehouses do not raise from the ground by themselves: they result from a collective sense of the problem and a global decision to create this organization. This paper proposes a simple method to design agents that can collectively expand their society with new organizations in order to cope with social problems. In particular, we want organizations emerge from endogenous social interaction, without requiring external triggers.

We demonstrate how to use this method in modeling the first stages of human development inspired by social theories $[2,3,7,8]$. These theories describe describe how tribal societies evolve into chiefdoms and states. According to them, one of the keys of social development lies in the emergence of social control organizations. For instance, the transformation of a tribe into a chiefdom is initiated by the increase of violent conflicts within the society, resulting from looser family bonds due to population growth. These fights are prevented by the emergence of a social control organization: a police, which lead by a chief.

In Section 2, we present the method to design our extensible societies. Then, in Section 3, we detail the social science theories and computer science models 
we were inspired by. In Section 4, we present the model of our simulation and we test its dynamics against social science expectations in Section 5. Finally, we conclude this article with discussions about applications and future work.

\section{$2 \quad$ A Method To Build Evolving Societies}

We define a methodology to build societies which can dynamically expand themselves with new organizations in order to cope with social issues caused by changes in the environment or within the society itself. Moreover, we want these organizations to be created from bottom-up: to emerge from individual perceptions and actions and without exogenous trigger. In this section, we illustrate our method using the first transition of the model as an example. In this transition a tribal society is confronted with an increase in the number of fights. This triggers the creation of a police-like organization to prevent them.

Organizations are defined by the triple (purpose, cost, effect). purpose determines the preconditions needed to create the organization. cost determines organizational costs. effect represents the influence of this organization on the society. In this bottom-up approach, one of the effects is the enforcement of individual behaviors $b$ aiming at solving the purpose. Organizations can also be used as blackboxes in using wide range of effects (e.g. costs 10 units of resource and reduces the fight variable by $10 \%$ ). Moreover, organizations may hold internal dynamics, in order to be able to tune their effect and their costs with regard to the environment and their purpose (e.g. an increase of the purpose leads to an increase of the organizational size).

The method relies on this sequence of steps: (1) A global problem which is observed by the individual variable $o \in[0,1]$ affects individuals (step 1). $o$ increases when the problem is frequently observed by an agent. Thus, $o$ collectively increases when the problem affects the collectivity (e.g. fights become frequent). (2) This observation is reported to the rest of the society via a social merging mechanism $m \in[0,1]$. $m$ increases when $o$ collectively increases. Various solutions exist to represent $m$, (e.g. a vote, a petition, a strike action). Simple computer-oriented solutions can also be selected (e.g. averaging o). (3) If the problem observed by $m$ is important enough to meet the purpose of the organization $O$ and the society can afford the cost of $O$ (e.g. feeding policemen), then $O$ is created (a police). The effect of $O$ is applied, enforcing a problemsolving individual behavior $b$ (e.g. the protection behavior). The indirect effect is the resolution of the problem and the cost to hire individuals performing $b$. (4) $b$ should reduce the problem triggering $o$ (e.g. violence decreases) and thus $m$ (e.g. people feel safer). Note that organizations can rely on $m$ as a performance indicator to determine if their effect should be amplified, reduced or kept stable while keeping the cost as minimal as possible. (5) Finally, $O$ is removed if the problem is do not occur (purpose and effect are both low) or if the cost cannot be afforded. A diagram of the organization creation process is presented in Figure 1 using the MASQ formalism [9]. 
In this method, the emergence of an organization is endogenous: it is the consequence of agent-level parameters. There is no external trigger that directly creates the organization.

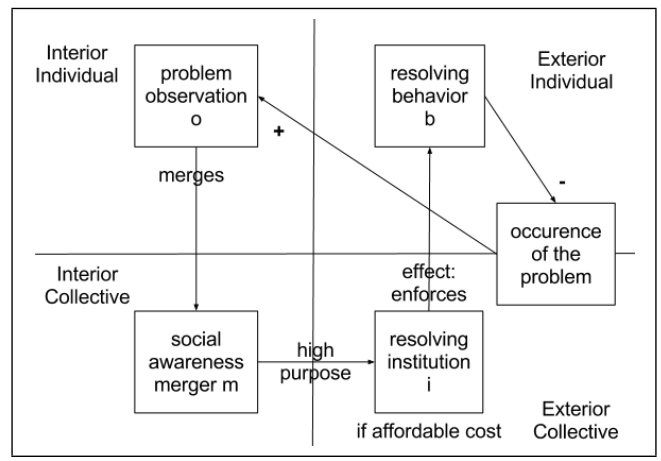

Fig. 1: Conceptual diagram of the organization creation process using the MASQ formalism [9]. Interior Individual represents the information process done within the agent, Interior Collective represents the shared beliefs amongst the society, Exterior Individual is what can be observed in the environment and Exterior Collective is what is globally observable from the simulation at the collective level.

\section{Related Work}

Evolving Societies: Social Sciences Perspective Several authors $[2,3,7,8]$ formulated theories on the long-term evolution of human societies. According to them, societies evolve through generic stages, each stage being characterized by the creation of a new population-control organization that tackles issues caused by shifts in population size. Thus, this theoretical framework fits the type of models we want to build with our method. Our model is mainly inspired by Diamond's theory because it proposes well-described transitions (problem and solutions) at the individual level. Nonetheless, depicted stages (band, tribe, chiefdom and state) are relatively similar across theories. As bands and tribes have similar organizational entities, with the difference that bands are smaller and nomadic our model starts with the tribe stage.

Each social stage corresponds to a social paradigm, which fits a given population size. For instance, a society with 100 members and another one with 10000 members cannot work the same way, for obvious practical reasons (e.g. resource management, decision making). Theories describe that the social stage changes when the population reaches the population-control limits of its society in creating a new population-control organization. Otherwise, if the population is too big for the social stage, social issues prevent the population to grow further. If the population is too small, some population-control organization become useless and are removed.

Tribes are groups from 80 to a few hundred people. The population is small enough to keep short the inter-individual familial distance. This kinship is a natural population-control mechanism favoring mutual exchanges and preventing 
fights to occur even in difficult conditions. There is no formal power centralizing information and decision which is reached through consensus.

When the population grows, the average familial distance between individuals increases. Consequently, the familial conflict resolution process fails to prevent fights. Moreover, reaching consensus on decision making and resource management becomes harder with numerous inhabitants. These limitations trigger the social transition from tribe to chiefdom.

Chiefdoms are groups up to thousands of individuals directed by a leader or a family of leaders. Leaders control power and information. Thus, this organization solves the scaling issue of the tribal consensual decision making. Moreover, leaders centralize and redistributes resources via a taxation system. This system allows leaders to feed specialists. These specialists can produce luxury goods for leader (inducing a cost of resource) but also control the population (policemen) preventing fights between unrelated individuals.

At the chiefdom stage, the management of critical information and decision making is made only by leaders. When the society grows (above 50.000 individuals), leaders become overwhelmed by information and decisions. They have to delegate a part of their power to subordinates. But, without tight control, subordinates tend to take over the leader.

To prevent this situation, the propagation of cultural values (e.g. law, norm, religion) that supports the leaders and their system have been proven successful. Such a culture generally contains rules and processes and decision and some ideology grouping members of the society within a moral circle. Thus, instead of obeying a leader, individuals act according to rules (decentralizing the decision process) for to defend the values carried on by the ideology. This culture is indirectly spread amongst the population via what we call Cultural Harmonizers $(\mathrm{CHs})$ whose activity implies spreading the culture (e.g. priests, professors, itinerant storytellers). A society maintaining a strong culture with $\mathrm{CHs}$ is at the state level.

Table 1 provides a summary of the observed specificities for each social stage.

Table 1: Social patterns generally observed for each social stage (based on Diamond's theory [3])

\begin{tabular}{|c|c|c|c|}
\hline & Tribe & Chiefdom & State \\
\hline Number of people & Hundreds & Thousands & Over 50.000 \\
Settlement pattern & 1 village & 1+ villages & Many villages \\
Decision making & "Egalitarian" & Centralized & Centralized \\
Leadership & Big man & Hereditary & Organizational role \\
Force and information monopoly & No & Yes & Yes \\
Conflict resolution & Informal & Centralized & Law, judges \\
Exchange & Reciprocal & Redistributive (tribute) & Redistributive (taxes) \\
Indigenous Literacy & No & No & Yes \\
\hline
\end{tabular}

Each stage is the consequence of the emergence of a new organization: a police at the chiefdom stage and a $\mathrm{CH}$ organization at the nation stage. Thus, these theories propose a framework that matches the purpose of our methodology. 
Evolving: Computer Science Models Previous research in computer science has also investigated the replication of emerging phenomena observed in social reality, while keeping low agents simple. The most famous ones being Sugarscape [5] and New Ties [6]. Sugarscape [5] introduces a very simple grid-like environment and simple agents with simple rules. But, in adding slight variations on top of this environment and agents (like reproduction, fight and trade), Epstein and Axtell were able to replicate a wide range of observed social phenomena (e.g. genetic selection, global wars, real-world market behaviors). New Ties [6] aims at building an artificial society with socially high mental and linguistic capabilities, but with simple agents and simple environment. The goal of New Ties is to build autonomous entities, living and adapting to the world due to learning. Moreover, these agents are capable to produce their own culture and share it with other agents. The research objectives of New Ties aim at a better understanding of culture formation and linguistics. In this article, our research differs from them in the sense that we aim at building societies capable of dynamically expanding themselves with explicit institutions, but still in their bottom-up approach.

\section{A Model for Evolving Societies}

Our model consists of two main features: the first concerns environmental aspects (e.g. food, reproduction) and the second is focused on social aspects (human interactions). Environmental aspects represent food collection and reproduction. Well fed human agents reproduce. Thus, the society grows until the food input matches the survival costs of humans. If the food input is lowered, agents starve to death and the society becomes smaller.

Social aspects concern human agents social interaction. In particular, it introduces social issues caused by population scale changes. When the population is bigger than a family, family-unrelated agents are likely to fight with each other when the food is sparse. In this case, a police organization is created when the population feels globally unsafe. This leads the society to the chiefdom stage. Similarly, when the population increases, the loyalty towards the leader decreases. When the loyalty is too low, a $\mathrm{CH}$ organization is created to keep high loyalty, leading the society to the state stage. As the chiefdom stage arises before the state stage, state is also chiefdoms. In the following, the model is presented in more detail using the SugarScape [5] formalism. This formalism splits the model in three sections: environment, agents and model rules.

\subsection{Environment}

The environment is similar to the Netlogo space: a bounded $\mathbb{R}^{2}$ space for agents on top of which food is represented by NetLogo patches (a $\mathbb{N}^{2}$ grid). Each patch has 3 states: (sterile, harvested, full). A sterile patch never changes state. A full one can be harvested by human agents, thus becoming harvested. A harvested cell can become full each round $(p=0.005)$. In our experiments, patches in a radius of 8 around a village are initially full. Others are sterile. 
All environments, even the simplest ones, introduce complex dynamics in the model (as shown in [4]). The most important environment-related aspect of this model to be aware of is that the food input is relatively constant each round and sublinear to the number of harvesters. As a rule, the gain for each additional harvester is marginal when the number of harvesters is above 20 .

\subsection{Agents}

Human agents Human agents are entities populating the world. They are described by the following variables:

$h p \in\left[0, \max \_h p\right]:$ hit points or physical condition. This value decreases when the agent is attacked. If this value reaches 0 , the agent dies. max_hp is set to 10 in the simulation.

energy $\in[0$, max_energy $]$ : remaining energy. If this value reaches 0 , the agent dies of starvation and decreases by 1 every round. An agent with less than max_energy/2 units of food is hungry (it cannot reproduce), otherwise it is well_fed. max_energy is set to 400. Modifying max_energy impacts the sensibility of the population growth to external conditions: the lower this value is, the more the population fluctuates between periods of richness and extensive storage followed by overgrowth and starvation.

$b a g \in \mathbb{N}$ : amount of carried food. It is filled when agents perform the harvest action and its content can be stored in the village.

culture $\in[0,1]^{n}$ : a vector representing the cultural orientation of the agent (e.g. values and practices [7]), inspired by the Sugarscape cultural model [5]. Values of this vector are abstract, but can be used to compare inter-individual cultural similarity using Euclidean distance. This vector changes randomly with time (cultural innovation, change in opinion and divergence) and is be shared amongst individuals via the harmonize_culture action or inter-individual communication. Thus, inter-individual cultural distance evolves with time: it increases with the number of inhabitants and decreases with cultural sharing. We empirically set $n$ to $50 . n$ impacts on the variability of individual culture, but this impact is relatively low when $n>20$. culture defines the loyalty $\in[0,1]$ towards the leader. The farther the distance of the agent's culture and the culture culture $_{v}$ of the village, the lower this agent is loyal. Formally, loyalty $=$ $1-\alpha$.distance (culture, culture $_{v}$ ) where $\alpha \in \mathbb{R}$ is an arbitrary constant to adjust the impact of cultural distance over loyalty. In our case $\alpha$ is 0.05 .

$j o b \in\{$ harvest, protect, harmonize culture $\}$ : the activity to be performed during the "perform job" phase. A harvesting human moves to the closest food patch, collects it and drops its bag in the village. A protecting human is a member of the police. It is inactive during the "perform job" phase but increases the probability of the villagers to be under control in the "meet other humans" phase. Cultural Harmonizers ( $\mathrm{CHs}$ ) unify the culture of the village in performing the harmonize action. This action sets the culture vector of 10 human agents to the village culture culture $_{v}$. 


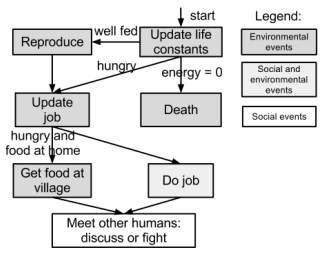

Fig. 2: The decision making process of a human agent

social_dissatisfaction $\in[0,1]$ : the dissatisfaction against the current society due to lack of physical protection. This value is reduced every round by $1 \%$ and increases by 0.2 (bounded by 1 ) when the agent is attacked.

Villages A village agent accommodates human agents and serves as deposit for their food resources. This agent is lead if a special organization (police or $\mathrm{CH}$ office) requires it. The leader establishes the social policy $S P . S P$ is a couple $\left(R_{\text {police }}, R_{C H}\right) \in[0,1]^{2}$. Each member of $S P$ determines the ratio of organizational jobs (police, $\mathrm{CH}$ ) per inhabitant offered to the population. Thus, $S P$ allows to tune the resource given to the organizations, in order to balance organizational costs and benefits.

food $\in \mathbb{N}$ : the amount of food stored by humans. Human agents storing their bag increase the value of food by the amount of their bag and set bag to 0 . When the village is lead, a portion (20\%) of the stored food is spoiled by leaders (to buy luxury goods). With this mechanism, we simplify the burden of sharing resources via exchange, trade or redistributive economy (which can also be achieved with our method, the redistributive economy being a pre-requisite of an organization for instance). Thus, the salary of organizational workers is the ability to eat from the common pot.

culture $_{v} \in[0,1]^{n}$ : the culture of the village. Without a leader, it is the average culture of its inhabitants, otherwise it is the culture vector of the leader.

harvest_tech_level $\in \mathbb{N}$ : the food production efficiency of the village (technological and environmental). When a human agent harvests some food, it puts harvest_tech_level units of food its bag. Empirically, the size of the society is correlated to the value of this variable. So, this variable is used in the experiments to determine the society size. Its values ranges between 10 and 300 .

\subsection{Rules}

Human rules Figure 2 shows the main steps of the decision making process of a human agent (individual behaviour).

Update life constants: energy is decreased (starving agents are removed) and culture is randomly tilted (one of its items is set to a random value) with a probability of 0.2 .

Reproduce: well-fed human agents can produce one child $(p=0.01)$. The energy value and culture are shared with the child. Other variables are set to default values. 
Update job: Periodically, humans change their job by updating the job variable. Harvest can be selected by anyone without restrictions. Institutional jobs (protect and harmonize culture), can only be selected if offered by the social policy $S P$. The job selection is simple: the agent tries first to get an organizational job. If none is available, it selects harvest. To avoid erratic behaviors, an agent is committed to a job for multiple rounds (randomly between 20 and 70 rounds).

Get food at village: The agent moves towards its village. If the agent is at its village and the food value of the village is high enough (bigger than max_energy), the human agent performs an eat action.

Do job: The human agent performs its job.

Meet other humans: each human agent engages in some social interactions with another randomly selected human agent from the same village. If these two humans are from the same family or under control of a loyal policeman, they discuss with each other. They can mix their culture vector if their cultural distance is close enough (according to the Axelrod cultural model [1]). This mechanism is important for our purpose: more agents lead to a more important cultural divergence. If the food is sparse, the two agents come into conflict. One of them is hurt ( $h p$ is reduced by 1 ) and complains about the lack of physical protection (social_dissatisfaction is increased by 0.2 ).

Two randomly-drawn agents belong to the same family with a probability $s /|v|$ where $s$ is the family size (set to 30 ) and $|v|$ is the village size. Two agents are under police control with a probability $P . L_{v}($ police $) /|v|$ where $P$ is the number inhabitants a policeman can control (set to 10$)$ and $L_{v}$ (police) is the sum of policemen loyalty. These mechanisms can of course be implemented in a different way. For instance, in a former version we represented explicitly family networks or policemen can patrol and defend their nearby space. The core property (whatever the complexity of its implementation) is that a population size increase triggers observable organizational failures (observed by $o_{1}$ and $o_{2}$ ).

Each round, each agent acts only once in the environment: during the "get food at village" or the "do job" phases. Available actions are: move: moves a distance of 1 . harvest: sets the local full patch to harvested and fills the agent's bag with harvest_tech_level units of food. harmonize: merges 20 items of the culture of an agent to culture $_{v}$ (for sake of simplicity we did not forced agents to be nearby to make this exchange occur). eat: the agent removes max_energy energy units of food from its village and sets energy to max_energy.

Village rules Village rules (Figure 3) represent orders given by chiefs (if any). They are split in 2 phases: organization creation and the social policy management $(S P)$. If no police organization is present, agents evaluate their desire for physical protection social_dissatisfaction. Each round, a vote is performed to determine if the police is required: agents approve if social_dissatisfaction $>0.7$. If a majority is reached, then a police institution is created and a leader (the human agent who survived the more battles) rules the village. Then, one of the human agents is hired as a policeman to perform the "protect" behavior. Similarly with the $\mathrm{CHs}$ office: agents evaluate the village loyalty. If the average loyalty is below 


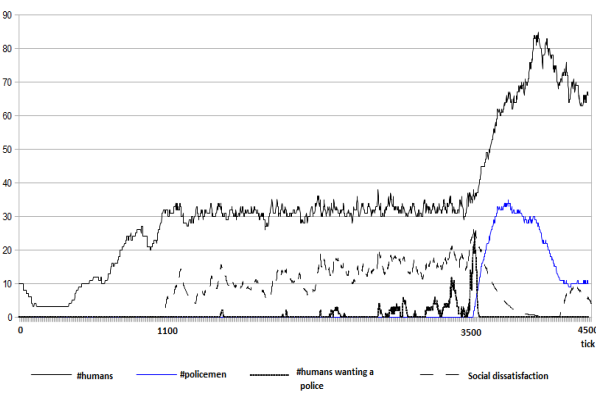

Fig. 4: The emergence of a police, leading the society to the chiefdom stage

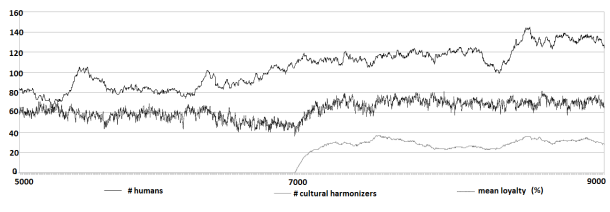

Fig. 5: The emergence of a $C H$ office, leading the society to the state stage

0.4 then the $\mathrm{CH}$ office is created. One agent is hired as a $\mathrm{CH}$, performing the "harmonize" behavior.

The policy management phase updates amount of resource allocated to each organization (their size) $S P_{v}$ according to the needs of the village. Leaders update $R_{\text {police }}$ to keep social_dissatisfaction below 0.5 and $R_{C H}$ to keep the loyalty above 0.7 . If $R_{\text {police }}$ or $R_{C H}$ is 0 , the need for the corresponding organization is gone and thus this organization is disbanded.

Our method represents the police with organization $O_{1}$. purpose $e_{1}$ is to prevent fights, cost $_{1}$ is the cost for feeding policemen and effect $t_{1}$ is policemen action. The local observation $o_{1}$ is social_dissatisfaction, the social merging mechanism $m_{1}$ is the vote and the individual behavior and $b_{1}$ is the "protect" behavior. $O_{2}$ represents the $\mathrm{CH}$ office organization with purpose $_{2}$ to raise loyalty $\left(o_{2}\right)$, cost $_{2}$ is the cost for hiring $\mathrm{CHs}$ and effect $t_{2}$ is the action of $\mathrm{CHs} . \mathrm{m}_{2}$ is the average of $o_{2} . b_{2}$ is the "protect" behavior. Both costs and effects of $O_{1}$ and $O_{2}$ are tuned using $m_{1}$ and $m_{2}$.

\section{Experimentation}

Our model aims at illustrating our method on a real-case inspired setting. In addition, we internally validate our model in showing that macro and macro behaviors of the model are consistent with the ones from the theories. Of course, this validation is not a "proof" that theories are correct.

What happens during the transitions? In this experiment we describe how societies change stage and create new social control mechanisms. To this extent, we increase the population capacity of the society in exogenously improving life conditions with time (harvest_tech_level increases by 1 every 40 time units).

Figure 4 shows how the police organization is created, triggering the transition tribe-chiefdom. From time unit 0 to 1100, the population size is below the family size. Thus, even when the food is sparse, agents are not attacking each other. From time unit 1100 to 3000, some fights occur and are observed through $o_{1}$, but remains too sporadic to require the assistance of a police. During this period, life conditions improve, leading to more births and to more fights. Thus, $o_{1}$ increases during this period. The dissatisfaction reaches a climax from 3000 to 
3500 where more and more agents are highly dissatisfied $\left(o_{1}>0.7\right)$ leading to an increase of $m_{1}$. When $m_{1}$ reaches $50 \%$ of the population (tick 3500), the police organization $\left(O_{1}\right)$ is created. Due to an initial high average dissatisfaction the organization is allocated an important amount of resources. So, many humans agents are hired to perform the protect behavior $\left(b_{1}\right)$. This behavior reduces the number of fights $o_{1}$, reducing in turn the overall dissatisfaction $m_{1}$. Note that the organization becomes eventually tuned for its purpose: after an initial overgrowth, the ratio of policemen is reduced to keep the social dissatisfaction low without extra costs.

Figure 5 shows the emergence of the $\mathrm{CH}$ organization, leading the society to the state level. Due to a population increase, the traditional communication mechanisms are not sufficient for the population to globally agree leading in turn to a reduction of the average loyalty. The average distance between individual's culture and culture $_{v}$ tend to increase with the population size. The individual disagreement with the village culture is observed with $o_{2}$ and is collectively averaged with $m_{2}$. In time unit 7000, the society observes a global disloyalty $\left(m_{2}<40 \%\right)$ triggering the creation of a $\mathrm{CH}$ organization $\left(\mathrm{O}_{2}\right) . \mathrm{O}_{2}$ hires $\mathrm{CHs}$, who spread culture, reducing the average distance between individual's culture and culture $_{v}$ and thus raising the average loyalty to 0.7 with the "harmonize" behavior $\left(b_{2}\right)$. These experiments show the adaptability of our model in dynamic environments: organizations can be created, kept and disbanded on the fly to fit in a dynamic environment and society.

As a general observation, when harvest_tech_level increases, society size increases as well as the pressure on the society to evolve to the next stage. The first transition can occur when harvest_tech_level reaches 80 and the second when harvest_tech_level reaches 150 . Thus, changing harvest_tech_level update rate only influences the amount of time needed by the society to evolve.

In the current model, harvest_tech_level is modified exogenously, because we aim at observing the emergence of social control institution to illustrate our method of problem-tackling organizations. Nonetheless, we experimented more complex models (not presented here for space considerations) that include endogenous growth of harvest_tech_level . In these extensions, individuals perform research actions through a third organization dedicated to research (artists, as described in [3]). We obtained similar results as those obtained here (the same transitions) even if dynamics induced by research organizations influence other factors directly (e.g. the population growth speed) and indirectly (e.g. other organizations).

Some other observations made by theories at the macro level also appear in this simulation. For instance, a society in an "obsolete" stage cannot grow due to social issues. Thus, the emergence of a new stage generally triggers a demographic explosion. Similarly, the problem-solving organization is generally overemphasized during its first steps due to extremely high demand and is then reduced to a more reasonable size. Moreover, if the population is reduced, less fights and more loyalty are observed. If an organization achieves its purpose in spite of being empty $R_{i}=0$, then this organization is disbanded. 
Skipping stages? Another important property of the human societies described in [2,3] is that even if life conditions are good enough, a stable state cannot be built directly from a tribe, while skipping the chiefdom stage. The chiefdom stage brings the necessary social control organization to prevent the society to collapse. They use this type of examples to describe why societies evolve through well-defined stages. We simulated this "what if" question with our model with the following hypothesis: can a society in a wealthy environment expand to the nation stage (build a $\mathrm{CH}$ organization) and remain sustainable without reaching the chiefdom stage(build a police organization)? To this extent, we run our model in setting harvest_tech_level to 300 (or any higher value) and preventing the police organization to be created (agents never vote "yes" for a leader in the village phase).

In these conditions, the society initially starts by growing, until reaching the point where the loyalty to the village becomes low. Then, the $\mathrm{CH}$ organization is created and the population keeps growing until the resource consumption meets the available resources. Then, individuals start fighting. Since no police exists to prevent combats, human agents fight until killing each other. Thus, the society collapses until reaching approximately the size of a family and is then limited at this point. This reduced size eventually triggers the removal of the $\mathrm{CH}$ organization, since the tribal communication mechanism is sufficient to keep the loyalty towards the village high enough. Thus, the society has returned to the tribal stage. Consequently, our model matches the predictions made by the theories at the collective level: each societies have to evolve one stage after the other and in the correct order in order to remain sustainable.

\section{Conclusion and Future Work}

In this paper, we presented a method to build societies capable of extending themselves with organizations solving collective issues. These organizations emerge from bottom up, with a mechanism which is internal to the society and based on the agent's local perceptions. Moreover, these extensions can be created, strengthened and removed on the fly, making the society capable of adapting to environmental and social changes. The method consists in merging the local observations of the issue amongst the society. This merging allows the society to become aware of the situation and then to build the appropriate organization in order to solve the issue.

We tested this method in modeling the first stages of human societies. These stages are characterized by the occurrences of two organizations, the police and the cultural harmonization (e.g. temples or schools) organizations, solving societal growth issues. The modeling and the implementation of the dynamic creation of organizations are straightforward from the method. Moreover, our artificial societies display similar behaviors to those of societies described by human development theories (e.g. skipping social development stages eventually leads to a social collapse). 
Future work will focus on extending the model and the method. The model can be extended to include a more credible influence of the culture, as the one described by Hofsede et al. [7]. The model is also being refined in order to make the village emerge from bottom up on a grid. We expect that our methodology is sufficient to raise similar emerging organizations.

The method can also be refined into a logic, allowing to express dependencies for instance. Consider for example the need for bread in a society. Making bread requires flour, the flour requires weat and a mill. Thus, this logic would describe how to make an economy emerge from scratch and simplify its design. The outcome of this research has interests in various fields, like games (the previous example is drawn from Settlers II), simulation of complex societies and self-organizing systems. This logic would be capable to consider multiple organizational responses for the same issue, with different costs and benefits (e.g. official police, unofficial night brigade, civic education at school). So, this logic allows to build societies capable of growing several combinations of organizations in keeping into account its environment.

\section{Acknowledgements}

The first author wishes to thank Melania Borit for her feedback while writing this paper.

\section{References}

1. R. Axelrod. The Dissemination of Culture: A Model with Local Convergence and Global Polarization. Journal of Conflict Resolution, 41(2):203-226, 1997.

2. D. E. Beck and C. Cowan. Spiral Dynamics: Mastering Values, Leadership and Change. Wiley-Blackwell, 1996.

3. J. Diamond. Guns, Germs and Steel. Vintage Books, 2005.

4. V. Dignum and F. Dignum. Exploring congruence between organizational structure and task performance: A simulation approach. In Coordination, Organization, Institutions and Norms in Agent Systems I, Proc. ANIREM05/OOOP05, volume 3913 of LNAI, pages 213-230. Springer, 2006.

5. J. M. Epstein and R. Axtell. Growing artificial societies. The MIT Press, 1996.

6. N. Gilbert, M. Den Besten, A. Bontovics, B. G. W. Craenen, F. Divina, A. E. Eiben, R. Griffioen, G. Hévízi, A. Lõrincz, B. Paechter, S. Schuster, M. C. Schut, C. Tzolov, P. Vogt, and L. Yang. Emerging Artificial Societies Through Learning. Journal of Artificial Societies and Social Simulation, 9(2):9, 2006.

7. G. Hofstede, G. J. Hofstede, and M. Minkov. Cultures and Organizations: Software of the Mind, Third Edition. 2010.

8. A. Johnson and T. Earle. The evolution of human societies : from foraging group to agrarian state. 2000.

9. T. Stratulat, J. Ferber, and J. Tranier. MASQ: towards an integral approach to interaction. Mind, 2:813-820, 2009. 